\title{
A Phenomenological account of Emotional Experiences in Depression among Iranian patients
}

\section{Abstract}

Emotional experiences play a central role in relationships with others. The aim of this paper is to study the manifestation, and phenomenological significance of emotions in depression, as they occur in the domain of interpersonal relationships, and the way they are influenced by culture. Adopting a phenomenological perspective, and using Sartre's accounts of interpersonal relationships and the significance of emotions, I offer an account of emotional experiences in depression among Iranian patients. As compared to those in the UK, Iranians complain about a range of different emotional experiences in depression. These include the replacement of guilt with aggression, and the desire to isolate oneself from others, as opposed to feeling lonely, as is seen in the UK. These differences, as I show, can be accounted for through a) phenomenological considerations of the kind of experiences we as human beings go through, and b) cultural norms and frames of thought which play a role in the significance of such experiences and their interpretations.

Keywords:

Depression, Emotions, Interpersonal Conflict, Iran, Cross-Cultural Phenomenology 


\section{Introduction}

Cross-cultural variation in mental disorders, including depression, have been subject of various studies, mainly in medical anthropology and cross-cultural psychiatry. Seminal works by Arthur Kleinman on depression in China (e.g. Kleinman, 1982) are noteworthy in this context, as is the collection of studies presented in Culture and Depression (Kleinman \& Good, 1985). Some works have also examined cultural variations in pre-morbid personality associated with depression (Ogawa, et al., 1992). Although there have been some studies on depression and dysphoric affect in Iran (e.g. Good, 1976, Good, et al., 1985, Behrouzan, 2016), the objectives of these differ from the study at hand. This paper, situated within this broader discussion, aims to offer a phenomenological account of some cross-cultural variations in experiences of depression. Here, individuals and their experiences are viewed as situated within a sociocultural context, and variations in experiences are analysed and accounted for given this cultural embeddedness.

Differences in phenomenology of depression between the UK and Iran were investigated via the use of a questionnaire. In Experiences of Depression (2015) Ratcliffe presents the findings of a study using a questionnaire completed by patients with depression in the UK. The same questionnaire (translated into Farsi) was employed to investigate the experiences of people with depression in Iran (See Appendix). Responses to a pilot study indicated that there were likely to be important differences in the ways UK and Iranian patients experienced interpersonal relationships, and some additional questions were added to the questionnaire to further probe the Iranian experience of interpersonal relationships in depression. It was initially planned to make the questionnaire available to Iranian patients online (as had been done in the UK study). However, it proved difficult to find a suitable online platform in Iran. To increase the number of responses, the questionnaire was also distributed in a University Clinic in Shiraz. A total of 43 responses were recorded (10 males and 33 females), 
13 of which were completed online, and the remaining 30 were completed by patients at the clinic. Five of the respondents had received diagnoses of other mental disorders as well as depression (two bipolar and three obsessive disorders). All numbered quotes included in this paper are taken from responses to this questionnaire.

Striking cross-cultural differences in experiences between Iran and the UK, relate to the manifestation of the breaking of the interpersonal bond in depression. The existential change in depression, influencing the way one views other people and one's relationship with them, plays a significant role in shaping the overall experience of depression. In this paper, I look at interpersonal experiences in depression as seen in Iran, and show how some elements of these experiences are different from those seen in the UK, due to cultural differences.

The most salient differences found in this context are, firstly, that whilst those in the UK complain of feeling lonely, Iranians talk about wanting to isolate themselves and 'escape' from other people. And secondly, while UK patients feel guilty, Iranian patients more commonly feel aggressive. I will suggest that these differences can be accounted for through cultural variations. In particular, cultural elements at play here are 1) the misconceptions of depression in Iran and the hostile attitudes arising from it, and 2) the collectivist nature of Iranian society and cultural norms of relations with others, which influence the emotional experiences between individuals.

With these cultural elements in mind, I will use Sartre's account of interpersonal relationships, and emotions, as the framework for understanding the dynamics of interpersonal conflict which, I argue, underlies the emotional experiences in depression. Sartre gives an account of the universal nature of our being and consciousness and as such, when it comes to culturally specific behaviours and patterns of thought and interpretation, his account will only provide guidance for framing the analysis, rather than providing a theory to be directly applied to the case at hand. I first set out the Sartrean theoretical background before turning to the 
emotional experiences among Iranian patients with depression, in the domain of personal relationships.

\section{Relating to others as persons}

Sartre (2003) maintains that at the heart of difficulties in interpersonal relations, is an inherent interpersonal conflict, arising from the way we see ourselves in relation to the Other. This conflict, he argues, arises from our way of being, and relating to others.

In Being and Nothingness (2003), Sartre observes that the fundamental element in our human consciousness is intentionality - the fact that our consciousness is first and foremost directed outwards and is consciousness of something, out there in the world. In our awareness of ourselves and the world, therefore, we are conscious subjects aware of other things that comprise our sense of the world in which we dwell, and these other things are always taken as objects of our consciousness, i.e. as things that we are consciously aware of.

However, this conception of oneself as inherently a subject, is fundamentally transformed in one's encounter with the Other. Through the recognition of the Other as a conscious being such as myself, i.e. as a person, I realise the possibility that as a subject, the Other can take me as the object of his consciousness. This recognition of the Other as a person like myself, Sartre claims, comes from certain emotions that I can only have in the presence of the Other, such as shame and guilt. Sartre argues that the very existence of these emotions, the very possibility of experiencing them, makes evident the essence of the Other as a person for whose consciousness I can be an object. In such a situation, I realise that the Other can see me, and this being seen by the Other, reveals me to myself anew. This is since I now see myself through the eyes of the Other, as I appear to the Other, as an object, and through the judgements that the Other can pass on me. "By the mere appearance of the Other, I am put in the position of passing judgement on myself as an object, for it is as an object that I appear to the Other" (Sartre, 2003, p. 246). 
The presence of others opens up new possibilities for me. Possibilities which rely on the recognition of others as persons, and which include those of meaning-making, the experiencing of certain emotions, and understanding of oneself and the world where one dwells. In Sartre's terminology, in the presence of the Other, my Being for myself - being-for-itself - is transformed into the mode of being of things - being-in-itself -, in the eyes of the Other. This transformation is crucial for me to realise my being fully, since it makes possible a more complete knowledge of myself, which exist only in the presence of the Other. It is because of this crucial transformation and the new possibilities brought about by it, that Sartre argues that our being as humans is essentially social; it is through our relationships with others that our being is made complete: "I need the Other in order to realize fully all the structures of my being" (Ibid.). And it is thus that our being, as a For-itself, is inherently, and essentially, a being-forothers.

This transformation, however, also gives rise to conflict. This arises due to the unfamiliar nature of myself in the face of the Other: the Other forces me to see myself in a new way, one which bears no resemblance to the image I hold of myself, i.e. as a subject with certain characteristics. This objectification of my being by the Other is naturally met with fierce resistance and leads to an unresolvable conflict in which both I and the Other attempt to establish our subject-ness by escaping the inevitable objectification of the other. This struggle is seen in all forms of relationships, as Sartre argues, from loving to hateful to indifferent, at the heart of all kinds of relationships remains an unresolvable and necessary conflict. This is since in all instances, different in appearance as they might be, the struggle to save one's subjectivity from the look of the Other will be present.

Everything which may be said of me in relations with the Other applies to him as well. While I attempt to free myself from the hold of the Other, the Other is trying to free himself from mine, while I seek to enslave the Other, the Other seeks to enslave 
me. We are by no means dealing with unilateral relations with an object-in-itself, but with reciprocal and moving relations. (Sartre, 2003, p. 386)

This conflict is important, since it enables one to see an image of oneself that would otherwise remain concealed. Additionally, this conflict generates certain emotions, experience of which would be impossible without the presence of the Other. To understand this importance and the way these emotions arise, it is important to have an account of emotions themselves.

\section{A Phenomenological Theory of Emotions}

In Sketch for a Theory of the Emotions, Sartre offers an outline of a phenomenological account of emotions, whereby emotions are significative of the human reality, of the whole of one's consciousness; arguing that emotion is "an organized form of human existence" (Sartre, 1962, p. 28).

Emotions, like other acts of consciousness, are intentional and are directed towards the world. In experiencing fear, one is afraid of something out there in the world, and in experiencing love, the emotion is directed to a certain object or person out there in the world. Emotional consciousness, therefore, is consciousness of the world, and because of the relational nature of emotions, the subject and object of an emotion are "united in an indissoluble synthesis" (Ibid., p. 57). Emotions, Sartre argues, form a specific manner of apprehending the world in which we dwell. For example, take the sense of irritation at one's inability to solve a problem. Whilst solving the problem, one is aware of different things out there in the world that contribute to the problem, or to the attempt at solving the problem. The irritation itself is a way the world appears to one. The emotional experience of irritation is both a reaction to the world, and a way of understanding and apprehending the world. It can be seen, therefore, that our emotions are entangled with the world and the way it appears to us. Thus, emotions are always significative of our human reality: the way we find meaning in the world where we dwell, and the way in which we see the world itself. 
Emotions, Sartre argues, are transformative of the world we live in, since in their presence, we see the world in a new way and therefore are presented with different possibilities offered by this new world. This transformative nature of emotions, rooted in imaginative consciousness (Sartre, 2004), is what differentiates emotional ways of apprehending the world from other ways of doing so. We perceive our environment "as a complex of instruments, a medium in which, provided we know certain rules or techniques, we can manipulate people and things so as to achieve certain ends" (Fell, 1965, p. 15). This perception of the world is made possible due to our intuitions about the nature of the world as orderly, as "ruled by deterministic processes" (Sartre, 1962, p. 63). It is only based on our presumptions that the world is regular, that we assume certain actions would guarantee certain ends. In emotions, however, through the power of imagination, this perception is transformed:

When the paths before us become too difficult, or when we cannot see our way, we can no longer put up with such an exacting and difficult world. All ways are barred and nevertheless we must act. So when we try to change the world; that is, to live as though the relations between things and their potentialities were not governed by deterministic processes but by magic. (Sartre, 1962, p. 63)

Sartre provides a simple example to clarify how emotional consciousness and behaviours transform our understanding of the world and therefore our space of possibilities. Take the situation in which I reach to pluck a bunch of grapes, but am unable to do so as they are beyond my reach. In response, "I shrug my shoulders, muttering: 'they are too green"” (Ibid., p. 65). Sartre argues that conferring the quality of being 'too green' on the grapes, acts as a substitute for the act which I intended or desired, but were unable to complete. In other words, the attractive quality by which they were presented to me - as 'ready for gathering' as Sartre puts it -, becomes intolerable when the possibility presented to me cannot be actualised. The tension created through this inability to actualise a possibility is resolved when I confer a new quality 
onto the grapes; i.e. their being 'too green'. Now of course, one cannot confer this new quality chemically, but rather through "putting on the behaviour of disrelish" (Ibid., p. 66). It is in such a way that I confer the quality onto the grapes imaginatively, and it is thus that emotions come about: "In this case the comedy is only half sincere. But let the situation be more critical; let the incantatory behaviour be maintained in all seriousness: and there you have emotion" (Ibid.).

Emotions, therefore, are like other acts of consciousness, purposive: they are a way of acting, with imagination underlying the 'magical' transformative power of emotions. The transformation of one's perception of the world, from a deterministic and pragmatic one, into a magical, or imaginative one, enables one to see new possibilities, new ways for achieving a goal, or new ways of apprehending the world and changes in one's space of possibilities. This is since, in imaginative transformation, the perceived object is transformed so as to conform to our desires towards the object. It is in this sense that our emotions remake the object in imagination, and it is through this remaking that new possibilities arise: the deterministic nature of the environment no longer presents itself as a limiting condition, since the new quality projected onto the world (or a certain object in the world), presents to one's consciousness a new way of apprehending the world. This new apprehension, then, presents new potentialities, new possibilities, and new ways to act upon one's desires, by directing one's emotional consciousness to the world. Therefore, in having an emotion directed to the world, or something within it, we are acting upon the world, by projecting our desires onto it, and in so doing, we find a new way of apprehending the world. Given this relational nature, Sartre's theory offers a way of understanding emotions, as significative of our being-in-the-world: "Emotion is a sign of the conscious interpretation man puts on his experience" (Fell, 1965, p. 51).

\section{Empathy and Unhomelikeness}

So far the concern has been with the nature of emotions and emotional consciousness. Understanding emotional experiences in depression among Iranians requires brief discussion 
of another notion, namely moods. In this discussion, I follow Heidegger's account of moods; as an attunement to the world, colouring the way everything appears to one. Unlike emotions, moods are not directed towards a specific object, are not thought of as ways of acting upon the world, and rather "form of their own accord, as something we cannot forcibly bring about, but into which we slip unawares" (Heidegger, 1995, p. 59). Moods disclose the world we inhabit, defining our being 'there' in the world, and thus form the backdrop of our experiences of beingin-the-world, including emotional experiences. ${ }^{1}$

Using this notion of moods, Svenaeus (2000a, 2000b, 2011) develops his account of unhomelikeness, as the defining mood in illness, characterised by a sense of unfamiliarity in the world and one's body. Despite the all-encompassing nature of this mood in illness, its manifestations can be different in different illnesses, and in particular domains of illness experience (compare sense of unhomelikeness due to impared physical ability, to one due to altered perception of the world in affective disorders). In the case of the experiences of depression in Iranian patients, part of this mood of unhomelikeness is brought about through misunderstandings and misconceptions of depression. These arise in part from folk beliefs and theories about the nature of illness more generally, causing a sense of unfamiliarity in the (social) world the patient inhabits. In Iranian folk understanding, as I argue at length elsewhere (Mirdamadi, forthcoming), illness is construed as essentially a physical phenomenon, with at least some physical symptoms and manifestations. Thus, the understanding of depression as primarily a non-physical illness, without familiar observable physical symptoms such as tissue damage, is lacking. Such misconceptions of depression, often lead to judgements and attitudes that can be stigmatising, and perceived as lacking in empathy, thus creating a sense of

\footnotetext{
${ }^{1}$ There are similarities between moods defined as such, and existential feelings (e.g. Ratcliffe 2008 , 2015), yet a full comparative account of the two notions is beyond the scope of this paper. For consistency and precision, I follow Svenaeues in calling unhomelikeness a mood, acknowledging the parallels with existential feelings.
} 
unfamiliarity in the world for depressed individuals. A mood of unhomelikeness thus defined, and the perception of a lack of empathy on the part of others, forms the background of emotional experiences in depression among Iranians.

In giving an account of empathy, Ratcliffe argues that underlying the ability to empathise, is having "a kind of 'openness' to experiential differences" (Ratcliffe, 2015, p.238). It is not the case that in order to empathise, one would necessarily have to experience what another person is experiencing, i.e. moving from the second-person to the first-person to access a certain experience, giving rise to a "phenomenological isomorphism between the two parties" (Ibid. p.239), as is suggested in various theories of empathy. ${ }^{2}$ Rather, what is important is to establish a connection through understanding, whilst acknowledging the phenomenological differences between experiences. This 'openness to difference' in experiences, would be manifested in one's manners in relation to the person having the experience, in the form of curiosity to understand, for example in asking certain questions (or not asking others), being attentive to the other's needs, etc. Although it is noted that acknowledgment of the phenomenological differences in experience requires some grasp of the other person's experiences, Ratcliffe maintains that "this can remain vague, ambiguous, indeterminate, without amounting to a failure of empathy" (Ratcliffe, 2015, p. 239).

As such, the central element of empathy is an openness to understanding. The depressed patient who seeks to be empathised with, is seeking others, as a society but also as particular Others, to understand, or be willing to understand, her experiences in depression. Faced with judgements and attitudes arising from misconceptions of her illness, dominant in the Iranian society, the depressed patient feels that she is not being empathised with.

\footnotetext{
${ }^{2}$ Most prominent example of such theories is empathy as simulation, e.g. Stueber, 2006; Goldman, 2006, and similarly mirror or reconstructive empathy, seen in e.g. Goldman, 2011; De Vignemont, 2010
} 
\#14 - I felt like they didn’t understand me/empathize with me.

\#6 - When I'm depressed I feel like I don't have an emotional connection with anyone. Nobody can be helpful with their presence.

\#2 - I feel like they do not have the capability (tavānāyi) to understand at all.

And it is this sense, which creates and/or exacerbates the mood of unhomelikeness in the world; a sense of not being at home with the world and people within it.

Furthermore, on this account, empathy is construed as an attitude taken towards one. Feeling empathised with, therefore, would mean the recognition of this attitude in the Other. This recognition requires an ability to 'connect' with other people, and it is this ability which, because of the existential change and mood of unhomelikeness, involved and dominant in depression, is impaired: the very possibility of attaining and maintaining this interpersonal connection is lost in depression. The mood of unhomelikeness, already dominant due to the social misunderstanding of depression, further dominates the interpersonal relations. This is such that a depressed person is unable to identify in others the empathetic attitudes directed towards her, even in cases where such attitudes do indeed exist. This inability further gives rise to, and strengthens the central feeling of not being empathised with, or not being understood, which creates the backdrop of emotional difficulties in interpersonal relationships.

That this mood of unhomelikeness, arising from the socio-cultural sphere, adversely affects the interpersonal domain, is not surprising, since our encounters with others in the social setting influence our experiences, expectations, and attitudes in the interpersonal sphere. Inevitably, the general understanding of depression, and the attitude taken towards it in the social domain, are not distinct from those found in the interpersonal sphere, rather they influence one another, since the norms and attitudes held by the society represent the attitudes of individuals to a certain extent, and the individuals within the society have the power to 
change the norms of the society. For the depressed person, the lack of understanding in the social domain creates the expectation of receiving the same behaviour in the interpersonal domain. This loop then carries within it the sense of unhomelikeness as the dominant mood colouring the experiences of interpersonal relationships.

\section{Iranian Patients' Emotional Experiences in Depression}

The interpersonal experiences of depression among Iranians, relies heavily on emotional experiences. In addition to feelings of lack of empathy and interpersonal connection, Iranians often talk about having negative emotions towards others, or receiving them from others. These emotional experiences, in addition to being important in themselves, give rise to specific experiences and behaviours, an account of which would be incomplete without considering the role of the underlying emotions. Sartre's account is especially helpful in analysing the totality of such emotions, experiences, and behaviours. First he claims that emotions are a way of acting upon the world, transforming the world by projecting one's conscious interpretation of one's experiences onto the world. In this sense, then, emotions and experiences form a meaningmaking loop: through our emotions we apprehend our experiences and the world, while our being-in-the-world and our experiences of it shape our emotions. Second, Sartre emphasises the relation between emotional experiences and relations with others; since our being-for and with others makes possible certain emotional experiences which give new meaning to our structure of being in the world generally.

I would argue that lack of empathy, as perceived by depressed patients, is the underlying foundation of other emotional experiences. As seen in Sartre's account of emotions, perception and imagination present the mechanism by which one comes to have a certain emotion. In the case of depression, the perceiving act is one of lack of empathy on the part of others. In reaction to this perception, one's imagination and apprehension of this perception, projected upon the world in emotions, is governed by unhomelikeness as the dominant mood in depression, 
defining the perceived space of possibilities and one's view of others and the world. It is in this dynamic that one fails to connect with other people: "a kind of interpersonal connection that many of us take for granted seems impossible, absent from the world" (Ratcliffe, 2015, p.218). In this absence, what is highlighted instead, is the constant and ever-present conflict between one and others, which, as will become clearer, has a defining role in the emotional experiences in depression among Iranian patients. As such, this dynamic offers an understanding of how the interpersonal bond breaks down in depression; rather than considered in isolation, this breakdown finds meaning when considered in its entirety with different connected elements, emotional, behavioural, and cognitive alike. Throughout the following account, it should be borne in mind that the attunement of unhomelikeness dominates the way one thinks and apprehends one's perceptions of others.

\#6 - I see what they do and their behaviours in a pessimistic/negative way. The routine (roozmarreh) behaviour of, for example, my parents become frustrating (asabi) to me.

The perceived lack of empathy from others, and the mood of unhomelikeness that results from it, lead patients to view everything the Other does in a negative way, eliciting negative reactions in the form of attitudes and judgements. Against this attunement, the possibility of positive apprehension is lost, giving rise to the emotions under question.

\section{Negative emotions}

Compared to the UK, problems caused by misconceptions of depression are more prevalent in Iran. This is reflected in the questionnaire responses; patients comment on the lack of understanding of others and the burden they feel to legitimise their depression as a 'real' illness, both in the social and interpersonal domains. In the social domain, such misconceptions can lead to stigmatising attitudes towards depression and those suffering from it. In the interpersonal 
domain, patients often complain of other people's inability to understand their illness experiences, which in turn demands explanations that they are not always equipped to give.

\#1 - I'm not in the mood for having contact [with others] ... everyone wants to know why I'm not the happy and smiling person I used to be and this makes me more isolated.

\#2 - The most important thing I can think of [is] the explanation as to why I sleep so much, why I cannot work, why I don't take part in family activities and why I have become so powerless (nā-tavān) without a physical problem.

\#33 - [others annoyed me] by saying ‘you don't feel well, why are you like this, you look unwell, the way you speak is different'.

What is noteworthy here is the fact that these expectations arise from the social misunderstandings and misconceptions of depression itself. It is not the symptoms themselves, such as lack of energy and fatigue, that are questioned, but rather the presence of these symptoms in the absence of a physical illness. The patients are asked to legitimise their illness in a way to conform to the pre-existing assumptions and conceptualisations of what constitutes an illness.

However, there is an undeniable difference between such judgments and expectations in the interpersonal domain, compared to the social sphere. In the interpersonal domain such judgments become personal. Here, the lack of understanding and empathy, is not interpreted as having to do with depression itself, or one's experiences. Rather, patients feel that they themselves are not being understood, and other people do not have the capacity to understand them, as a person.

$\# 16$ - I feel like others also are not in the mood [to deal with] me and ... I feel like they don't understand me. 
\#14 - I felt like they didn't understand me.

This personal nature of the perceived judgment, can be interpreted phenomenologically as the feeling of the Others passing judgements as to the kind of person that I am.

The perception that those close to one lack the capability to understand one, against the backdrop of the attunement of depression, forms the basis of one's relationships with others in depression. In this sense, then, one sees others as adopting a hostile view towards one. This perception of hostility, as an instance of the breakdown of the interpersonal bond, is seen both in the case of Iranian patients and those in the UK. "All that others are perceived to offer is a distinctively personal form of threat, which might be experienced more specifically as derision, dismissal, ridicule, condemnation, aggression, or shame” (Ratcliffe, 2015, p.220). These various manifestations of this perceived hostility are present among the Iranian patients as well, and they persist even in others' absence.

\#14 - My sister didn't apprehend that I had depression, she felt my aim was to get attention from the family.

\#25 - Others get upset and say that I'm trying to be classy.

\#17 - I feel like others ... also talk behind my back and speak (badly) about me.

\#24 - My family hates me.

\#30 - [Others'] encounter has had a large impact - their behaviour [with me and my depression] has been really bad.

What is clear here, is the perception of a wide range of negative attitudes, judgements and emotions directed to one in depression. This perception brings to the foreground the conflict between one and Other, formed here, due to the lack of understanding on the part of others. Although these may be merely perceived, coloured by the dominant mood of depression, this perception is a necessary step for the creation of an emotional response, as seen in Sartre's 
account. The perception of the world being a certain way, with one's possibilities limited accordingly, motivates an emotional response, which take the form of negative emotions towards others. These negative emotions can be accounted for through two separate but linked considerations. The first is the already mentioned theory of emotions: following the perception of negative attitudes and judgements directed towards one, and in the background of the sense of unhomelikeness which dominates one's thoughts and outlook to the world, certain negative emotions arise towards others. Secondly, in the context of conflict between one and the Other, through the perception of the Other passing judgements on one, seeing one according to these judgements and in a way unfamiliar to the individual, one feels objectified by the Other. As seen earlier, when faced with such feelings, one inevitably moves to re-establish one's subjectivity by taking the Other as the object of one's consciousness and one way of doing this is through one's emotional consciousness towards the Other.

\#21 - I dislike everyone and nothing seems good to me. I feel hatred towards my children and family and want to escape from them.

\#7 - Sometimes in depression periods I would really really hate my father.

\#31 - I dislike all others.

\#28 - My brain didn’t believe what anyone was saying, like a person who hears something from one ear and it goes out from the other ear [Persian expression].

It is important to recall here the importance of such emotions, as instances of action taken by individuals in depression, but also as representative of their experiences and interpretation of their perception of the world around them. The feeling of hatred towards others, as well as being a reaction to the perceived negative attitudes and emotions towards the individual, are representative of the way one interprets one's position among others in the world. Therefore, these emotions are also informative of the way one sees the world more generally. The feeling 
of being stuck and the general sense of dissatisfaction with life are echoed in the emotions towards others. Sartre acknowledges this interconnectivity of one's emotional consciousness, and sees it as an important pillar of emotional experiences as significative of one's being more generally. "The impossibility of finding a solution to the problem is apprehended objectively, as a quality of the world. This serves to motivate the new unreflective consciousness which now grasps the world differently, under a new aspect, and imposes new behaviour - through which that aspect is grasped ..." (Sartre, 1962, pp. 64-65).

\section{Aggression and Guilt}

One striking difference between the manifestations of depression in Iran, as compared to the $\mathrm{UK}$, is the absence of feelings of guilt, and the presence of aggressive behaviour towards others.

Feelings of guilt and shame are associated with being negatively evaluated, by oneself or the Other respectively, for failing to meet certain norms and standards of what is considered right or appropriate. Therefore, such feelings are regarded as 'moral' emotions (Wong and Tsai, 2007). Additionally, shame and guilt are instances of 'self-conscious' emotions, "because they require a concept of the self, or an ability to see the self as an object of evaluation" (Ibid. p. 210). I suggest that the Iranian conception of the self plays a central role in accounting for the absence of guilt among Iranian patients and its replacement with aggressive behaviour.

A crucial difference between Iran and the UK, is the place and value of interpersonal relationships. As a collectivist society (Hofstede, 2001; House, et al., 2004), Iranian culture places heavy emphasis on shaping and maintaining social relationships, where the aims and goals of the individual are thought to have lower priority than that of the collective group. This contrasts with the individualistic nature of the UK society, in which the aims of the individual take precedence over the goals of the group. One important consequence of this structural difference, is that the concept of self is construed differently. "Individualistic societies are best characterized by their conception of an independent self that is bounded, unique, and generally 
autonomous ... social behaviour is generally judged to be driven from within and attributed to an individual's internal attributes" (Goetz and Keltner, 2007, p. 158). By contrast, in (mostly) non-Western, collectivist cultures, "an interdependent self is seen as part of an encompassing social relationship, recognizing that one's 'self' is determined by one's relationship with others and the group" (ibid.). Individuals in collectivist societies, further, tend to attribute the causes of events in their lives, especially negative ones, to external sources, events, or people (Triandis, 2001; Carpenter, 2000). In other words, in explaining events in their lives, individuals from collectivist societies are more likely to see external factors, including other people, as responsible rather than themselves. Unlike the UK patients, who either see their own shortcomings as the cause of their depression, or are unable to identify a cause at all, Iranians often attribute the cause of their depression to a source independent of themselves.

\#27 - The unsuccessful marriage that I had and the hard responsibility of a life with children [caused my depression].

\#20 - Death of the dear ones has caused my depression, the pitying and humiliation because of this sadness has caused it. That I used to go to the cemetery and cry, and that others humiliated me for this caused my depression.

\#25 - I had a friend who betrayed me and I loved them so much I couldn't believe it and I wanted to kill them but I visited the doctor and I was immediately hospitalised.

When Iranians attribute the cause of their depression to another person, against the backdrop of the mood of unhomelikeness and negative emotions and hostility between the depressed individual and Others, then aggressive feelings can be expected.

Iranian and UK cultures also differ in the extent to which others can be expected to help a suffering individual. Iranians often do favours for one another, friends and strangers alike, without being asked to do so, for example putting up banners and streetlights to welcome a 
neighbour from a long journey, or congratulating them on an achievement. The expectation that others will always be there for one in need, to care for one, is central in the normal day-to-day relations with others. However, the lack of empathy perceived by individuals in depression, can be thought of as an instance of this expectation not being met. Consequently, Iranian depressed patients feel a sense of abandonment by others, as well as disappointment that their expectations are not met. This is seen in patients' complaints of others' reactions to their depression experiences, and form the basis of holding others - at least in part - responsible for the pain they go through. Such feelings are in direct contrast to the feelings of patients in the UK: whilst those in the UK talk about feeling as if they have become a burden on Others, Iranian patients talk about others being bored and tired of their depression. In other words, for those in the UK, feelings of resentment and responsibility are turned inwards towards oneself, in Iran such feelings are turned outwards towards others.

\#26 - I felt that others were also tired of my depression.

\#16 - Others get bored with my depression and always annoy/bother me.

Such way of reasoning, as a point of difference between patients in Iran and the UK, can in part explain the absence of guilt and presence of aggression among Iranian patients. Criticism and dissatisfaction directed towards oneself is more likely to translate into feeling guilt and wrongdoing. This is while, as seen in the Iranian case, criticism directed outwards to others, and their failure to be or behave in a certain way as desired by the patient, motivates an outward reaction in the form of aggressive behaviour.

The interpersonal conflict at the foreground of consciousness in depression also plays a role in generating aggression. As seen earlier, part of what the interpersonal experience in depression involves, is a sense of hostility one feels directed towards oneself by others. This hostility is seen in the perception of negative emotions directed towards one, in turn eliciting 
emotional responses. In such cases, where one feels that her sense of self is being threatened by the negative valuations and judgments, and especially where one does not see oneself responsible and thus resistant to guilt, aggression and aggressive behaviours are more likely to come about (e.g. Stuewig and Tangney, 2007). Feeling misunderstood, receiving judgments which bring into question the 'reality' of one's illness and one's motivations for 'appearing ill', can be perceived as an attack on one's self, which warrants reactions of anger and aggression. As seen in Sartre's characterisation, in relations with the Other one is constantly made to reassert oneself as a subject, in response to feeling threatened by the Other's objectification of oneself. Against the backdrop of the breaking of the interpersonal bond, one where lack of understanding, negativity and hostility define one's encounter with the Other, aggressive behaviour could be thought of as a strong attempt to reassert oneself in response to the perceived attacks of the Other.

\#20 - I become aggressive, I don't answer the phone and am not in the mood for [being with] others, or I tell them not to call me or to stay away from me.

\#6 - I get aggressive. I don't have the tolerance for the simplest disagreements or arguments.

\#7 - I'm either completely defeated (maghloob), or angry and aggressive, or quiet (sāket) and stagnant/tranquil/static.

\#25 - I would get angry really quickly and easily ... I can't be kind to my children, I can't go to my shop and I always want to commit suicide or kill the one who has betrayed me.

Due to the variety and interconnectivity of the different elements at play here, it is difficult to single-out one element as the cause of the presence of aggressive behaviour in Iran and its absence in the UK. However, what the analysis here shows, is that a multiplicity of cultural 
norms and expectations, the severity of problems in the interpersonal and social domains in depression, and the range of emotions in reaction to these problems together give a convincing picture of the cross-cultural difference. I would also argue that the totality of these problems can be seen in the frame of the interpersonal conflict, exacerbated in depression, from which the individual attempts to escape, when faced with its severity and the impossibility of finding a resolution. This conflict is the 'problem', as Sartre argues, that motivates one's imagination to conceive of different emotional and behavioural reactions. And as one perceives the conflict as more difficult to resolve, so the reactions grow in intensity to resolve it. In the case of Iran, however, due to the strong entanglement between individuals, as well as between the individual and the social groups and society, where the lines between private and public life, between one and the Other, are blurred, one is neither able to resolve this conflict, since against the depressive mood such a possibility is not even conceived, nor to escape from it. And it is in this dynamic, that the most prevalent and talked about difference between patients in the two cultures is seen, namely, voluntary isolation and escape from others.

\section{Isolation}

In addition to aggressive behaviours, another notable difference in interpersonal emotions in depression between Iran and the UK, is Iranians' solitary tendencies. Ratcliffe shows that those suffering from depression in the UK often talk about a sense of solitude and loneliness, one that goes hand in hand with a sense of estrangement from the world more generally. On Ratcliffe's account, this feeling of loneliness is closely tied with that of lack of empathy and not being understood: "there is a feeling that [others] do not understand, which could equally be described as a feeling that they are unable to 'relate to' or 'connect with' the depressed person" (Ratcliffe, 2015, p.202). As such, lack of understanding is construed as evidence of the breakdown of the interpersonal bond, which leads to the depressed person feeling estranged from others, overcome by a sense of loneliness and solitude. 
In the case of Iran, however, there is no talk of feeling lonely. Rather, Iranian patients talk about wanting to escape from others and to be alone. Two important notes should be made with regards to this difference. Firstly, that the state of isolation from others is not only not complained about, but represents a desired state of being. Secondly, despite the feeling of lack of understanding present in both contexts, this feeling does not amount to a sense of estrangement from others, in the form of willing, but being unable to establish an interpersonal connection with others, as it does in the UK. Rather, it seems that the very willingness to do so is lost. I would argue that both these elements can be accounted for through the interpersonal conflict which dominates one's relationships with others in depression, and the differences in terms of the kind of society and the kind of prevalent norms in which the individuals are thrown.

\#15 - I was always in a corner not talking to anyone [the phrase is 'being in myself'], I was hermitic (goosheh-gir), avoided gatherings, was not in the mood to be around anyone.

\#17 - I'm not in the mood to be in social gatherings with friends, and prefer more to be on my own/isolated/hermitic.

$\# 18-[\mathrm{I}]$ go back into my shell and stop interacting with others.

\#19 - When I'm depressed I don't want to talk to anyone or go anywhere.

\#23 - When I'm depressed I isolate myself and become hermitic ... I am not really in the mood for them [other people], because I like to be alone when I'm depressed. \#25 - I want to be alone, and have nobody around me.

\#27 - I am not even in the mood for myself and I like to be alone ... I'm not in the mood for anyone around me ... I'm not in the mood to be with others at all and only want to be alone. 
\#28 - [I am] hermitic, I stay away from social situations ... in most cases I isolate myself and don't want to go see anyone, or let any visitors to come and see me ... I don't take part in any social gatherings, be it a funeral or weddings, and I'm not in the mood for [being around] my children and am mostly just with myself and isolated.

As mentioned earlier, in the Iranian context, the conflict between one and the Other dominates one's frame of thought and one's relationship with others. The conflict, represented in the endless loop of not understanding, negative attitudes and emotions, and aggression, is seen to be unresolvable. In other words, due to the circle of negative experiences and negative thoughts and attitudes, the very possibility of re-establishing the personal bond, one that is constructive rather than destructive, is removed from one's space of possibilities. In this case, then, the only change in one's behaviour that could result in the reduction of the conflict and the negativity that comes with it, is to remove oneself from social interactions, i.e. to escape from the interpersonal relationships and the conflict they present.

Furthermore, given the emphasis placed on personal relationships in Iranian culture, as a collectivist society, the notion that escape is the only way to reduce interpersonal tensions becomes more plausible. The cultural importance attached to relationships can be seen in the fact that despite certain changes, living alone remains an unacceptable way of life for most Iranians. It is generally accepted that the best way of life is one that puts family at the centre, and this means that most people live with family members. A life separate from family is seen as a flawed and incomplete way of life. Ultimately, such norms mean that the only way one could have a personal space to oneself, is by retracting from the everyday way of life, which is defined by the constant presence of, and contact with other people. In such a scenario, one does not feel alone, as there is always an Other present and as has been seen, this presence often brings with it feelings of conflict. This ever-present nature of the interpersonal conflict forces one to try and retract oneself from the social situations. 
It should further be noted that the cultural norms of interacting with others and being a certain way in their presence, could add more pressure on the depressed patients and in turn add to the frustration already felt in interpersonal relationships. For instance, one is expected to visit one's family regularly, or be hospitable to anyone who comes to visit one, whether invited or not. The fact that depressed patients talk about being unable to fulfil such expectations, represents both an expression of inability in illness which legitimises their suffering, and a source of pressure that makes the case for escaping from the social interactions ever so stronger.

\#21 - I isolate myself and really don't like to interact with anyone, or if anyone comes to visit me I don't feel like being hospitable.

\#28 - I stay away from others and stay at home alone and don't have any relation with others, I don't even go to [visit] my father's house.

\section{Conclusion}

I have given an account of the personal relationships and how they are affected and altered in depression in Iran. In addition to giving a phenomenological account such difficulties, I have argued that the cultural norms, expectations, and way of life, have an undeniable effect on the manifestation and experiences of interpersonal difficulties. As I have noted, particular crosscultural differences play a role in shaping the different emotional experiences of depression. First, greater misconceptions of depression in Iran, underlying social judgements and attitudes, form the backdrop of emotional experiences in depression. Second, due to the collectivist nature of the society in Iran, depressed patients tend to attribute the cause of their depression to external factors and people. This in turn exacerbates the aggression felt towards others in depression. Lastly, cultural norms around one's way of life and conducting oneself in Iran, are essentially centred around the presence of other people and one's duties towards them. This emphasis influences the way Iranians attempt to overcome the negative interpersonal emotions and 
experiences, namely by isolating themselves and escaping from others. This differs from depressed patients in the UK, who often complain of loneliness in depression.

In this account I have shown that both a phenomenological understanding and an appreciation of cultural norms is essential for a holistic understanding of experiences in depression. I have used a Sartrean account of emotions and our relations with others to illuminate on the experiences of the interpersonal in depression - these include the ever-present conflict between I and Other, which comes to the foreground in depression and defines the emotional reactions to the presence and actions of Others. I have shown how a consideration of differing cultural norms can illuminate the differences in behaviours and symptoms of depressive patients, as seen in Iran and the UK. I have demonstrated that cross-cultural comparison of the interpersonal experiences of depression, would be incomplete without looking at both phenomenology and culture as they inform and shape one another, and ultimately the individual experiences and manifestations of depression. ${ }^{*}$

\footnotetext{
* I would like to thank Ebrahim Moghimi M.D., and Sepideh Ghorbani who helped in the acquisition of data from Hafez University Clinic in Shiraz.
} 


\section{Appendix 1 - Depression Questionnaire}

\section{Personal details}

Age:

Sex:

Place of residence:

Have you ever been diagnosed with depression? If so, please give details of your diagnosis and the type of treatment you have received.

Have you ever been diagnosed with any other mental illnesses? If so, please give details.

\section{Questions}

1. Describe your emotions and moods during those periods when you are depressed. In what ways are they different from when you are not depressed?

2. Does the world look different when you're depressed? If so, how?

3. Do other people, including family and friends, seem different when you're depressed? If so, how?

4. How does your body feel when you're depressed?

5. How does depression affect your ability to perform routine tasks and other everyday activities?

6. When you are depressed, does time seem different to you? If so, how?

7. How, if at all, does depression affect your ability to think?

8. In what ways, if any, does depression make you think differently about life compared to when you are not depressed?

9. If you have taken medication for depression, what effect did it have? 
10. Are there aspects of depression that you find particularly difficult to convey to others? If so, could you try as best you can to indicate what they are and why they are so hard to express.

11. What do you think depression is and what, in your view, caused your depression?

12. Who and/or what have you consulted in order to try to understand your depression? (E.g., medical practitioners, friends, books, internet sources, etc.).

13. How would you describe your relationships with others when you are depressed?

14. Has the reaction of others influenced your experience of depression? If so, how?

15. What distinguishes what you're feeling as depression, from other, more general forms of sadness?

16. Are there any positive aspects associated with your depression?

17. If there are important aspects of your experience of depression not covered by this questionnaire please describe them here.*

*Questions 1-12 and 17 were used by the research team at Durham University, for the Experiences of Depression study. The questions are available online and reproduced in publications following the study. 


\section{References}

Behrouzan, O. 2016: Prozak Diaries: Psychiatry and Generational memory in Iran, Stanford, Stanford University Press.

Carpenter, S. 2000: "Effects of Cultural Tightness and Collectivism on Self-Concept and Causal Attributions", Cross-Cultural Research, 34(1), pp. 38-56.

de Vignemont, F. 2010: "Knowing Other People's Mental States as if they were One's Own”, in S. Gallagher \& D. Schmicking (eds.), Handbook of Phenomenology and Cognitive Science, Dordecht, Springer, pp. 283-300.

Fell, J. P. 1965: Emotion in the Thought of Sartre, 2nd ed., New York, Columbia University Press.

Goetz, J. L. \& Keltner, D. 2007: “Shifting Meanings of Self-Conscious Emotions across Cultures: A Social-Functional Approach", in J. L. Tracy, R. W. Robins \& J. P. Tangney (eds.), The Self-Conscious Emotions: Theory and Research. London, The Guilford Press, pp. 153-173.

Goldman, A. I. 2006: Simulating Minds: The Philosophy, Psychology, and Neuroscience of Mindreading, Oxford, Oxford University Press.

Goldman, A. I. 2011: “Two Routes to Empathy: Insights from Cognitive Neuroscience”, in A. Coplan \& P. Goldie (eds.), Empathy: Philosophical and Psychological Perspectives, Oxford, Oxford University Press, pp. 31-44.

Good, B. J. 1976: "The heart of what's the matter: The semantics of illness in Iran", Culture, Medicine, and Psychiatry, 1(1), pp. 25-58. 
Good, B. J., Good, M.-J. D. \& Moradi, R. 1985: “The Interpretation of Iranian Depressive Illness and Dysphoric Affect”, in A. Kleinman \& B. Good (eds.), Culture and Depression: Studies in the Anthropology and Cross-Cultural Psychiatry of Affect and Disorder, Berkeley, University of California Press, pp. 369-428.

Heidegger, M. 1995: The Fundamental Concepts of Metaphysics: World, Finitude, Solitude, Bloomington, Indiana University Press.

Hofstede, G. 2001: Culture's Consequences: Comparing values, behaviors, institutions, and organizations across nations, 2nd ed., London, Sage Publications.

House, R. J. et al. 2004: Culture, Leadership and Organizations: the GLOBE study of 62 societies, London, Sage Publications.

Kleinman, A. 1982: "Neurasthenia and depression: a study of somatization and culture in China", Culture, Medicine, and Psychiatry, 6(2), pp. 117-190.

Kleinman, A. \& Good, B. (eds.) 1985: Culture and Depression: Studies in the Anthropology and Cross-cultural Psychiatry of Affect and Disorder, Berkeley, University of California Press.

Mirdamadi, M. Forthcoming: Cultural Variation in Experiences of Depression in Iran and the UK: A Phenomenological Investigation (Unpublished Doctoral Thesis), Lancaster University, UK

Ogawa, T., Koide, H. \& Bouderlique, J. 1992: “Cultural Variations in Premorbid Personality of Endogenous Depression: A Transcultural Study", Psychiatry and Clinical Neuroscience, 46(4), pp. 831-839. 
Ratclife, M. 2008: Feelings of Being: Phenomenology, Psychiatry and the sense of Reality.

Oxford, Oxford University Press.

Ratcliffe, M. 2015: Experiences of Depression: a Study in Phenomenology, Oxford, Oxford University Press.

Sartre, J.-P. 1962: Sketch for a Theory of Emotions, London, Methuen \& Co Ltd.

Sartre, J.-P. 2003: Being and Nothingness, London, Routledge.

Sartre, J.-P. 2004: The Imaginary: A phenomenological psychology of the imagination, London, Routledge.

Stueber, K. 2006: Rediscovering Empathy: Agency, Folk Psychology, and the Human Sciences, Cambridge(MA), MIT Press.

Stuewig, J. \& Tangney, J. P. 2007: "Shame and Guilt in Antisocial and Risky Behaviours", in J. L. Tracy, R. W. Robins \& J. P. Tangney (eds.), The Self-Conscious Emotions: Theory and Research, London, The Guilford Press, pp. 371-388.

Svenaeus, F. 2000: “Das Unheimliche - Towards a Phenomenolgy of Illness”, Medicine, Health Care and Philosophy, 3(1), pp. 3-16.

Svenaeus, F. 2000: “The Body Uncanny - Further Steps towards a Phenomenology of Illness", Medicine, Health Care and Philosophy, 3(2), pp. 125-137.

Svenaeus, F. 2011: "Illness as unhomelike being-in-the-world: Heidegger and the phenomenology of medicine", Medicine, Health Care and Philosophy, 14(3), pp. 333-343. 
Triandis, H. C. 2001: "Individualism-Collectivism and Personality”, Journal of Personality, 69(6), pp. 907-24.

Wong, Y. \& Tsai, J. 2007: “Cultural models of shame and guilt”, in J. L. Tracy, R. W. Robins \& J. P. Tangney (eds.), The Self-Conscious Emotions: Theory and Research, London, The Guilford Press, pp. 209-223. 